\title{
UM ESTUDO SOBRE 5S NA DISTRIBUIDORA DE PRODUTOS DE HIGIENE PROFISSIONAL
}

\section{A SS STUDY AT THE PROFESSIONAL HYGIENE PRODUCTS DISTRIBUTOR}

\author{
Carlos Cesar dos Santos - carlosgeek1994@gmail.com \\ Faculdade de Tecnologia (Fatec) - Taquaritinga - SP - Brasil \\ Gustavo José Caçador - gustavo.cacador.gc@gmail.com \\ Faculdade de Tecnologia (Fatec) - Taquaritinga - SP - Brasil
}

DOI: 10.31510/infa.v17i2.918

Data de publicação: 18/12/2020

\begin{abstract}
RESUMO
No ambiente econômico mundial, 5S é um tema de extrema importância e altamente inserido no mercado, principalmente para as empresas que enfrentam a alta competitividade e aplicam o conceito de qualidade total, querendo maximizar a satisfação dos clientes. Nesta visão, a busca pela eficiência eleva a condição da qualidade. A permanência da organização no mercado é uma necessidade cada vez mais cobrada quando se trata de concorrência industrial. $\mathrm{O}$ método $5 \mathrm{~S}$ é um processo que viabiliza a busca da eficiência no ambiente de trabalho, por meio de 5 conceitos a serem aplicados, através da mudança de comportamento. $\mathrm{O}$ artigo mostra as etapas de implantação do $5 \mathrm{~S}$ em uma distribuidora de produtos de higiene profissional, com o objetivo de melhorar os indicadores de desempenho, proporcionando assim a redução no tempo médio de espera de atendimento do telemarketing, redução das reclamações pelo tempo médio de permanência dos veículos para carregamento e recebimento, redução do erro de carregamento dos itens e assim proporcionando o aumento da satisfação dos clientes. Cabe observar que os objetivos do $5 \mathrm{~S}$ agregam, de fato, desde a distribuição de matérias-primas até a etapa final do processo. Os resultados indicaram bastante satisfação na prática do $5 \mathrm{~S}$, tentando manter o máximo de controle possível e significativo, indicando melhorias obtidas em relação a limpeza, autodisciplina, organização, utilização de saúde e higiene, diminuindo assim os desperdícios e as falhas, melhorando o clima organizacional, aumentando a produtividade e a motivação dos colaboradores.
\end{abstract}

Palavras-chave: Método 5S. Indicadores de Desempenho. Melhoria de Processos.

\begin{abstract}
In the global economic environment, $5 \mathrm{~S}$ is an extremely important topic and highly inserted in the market, mainly for companies that face high competitiveness and apply the concept of total quality, wanting to maximize customer satisfaction. In this view, the search for efficiency raises the condition of quality. The organization's permanence in the market is an increasingly demanded need when it comes to industrial competition. The $5 \mathrm{~S}$ method is a process that enables the search for efficiency in the work environment, through 5 concepts to be applied, through changing habits. The article shows the $5 \mathrm{~S}$ implantation steps in a
\end{abstract}


professional hygiene products distributor, with the objective of improving performance indicators, thus reducing the average telemarketing waiting time, reducing complaints due to the average length of stay of vehicles for loading and receiving, reducing the loading error of the items and thus providing increased customer satisfaction. It should be noted that the objectives of $5 \mathrm{~S}$ add, in fact, from the distribution of raw materials to the final stage of the process. The results indicated a lot of satisfaction in the practice of $5 \mathrm{~S}$, trying to maintain the maximum possible and significant control, indicating improvements obtained in relation to cleanliness, self-discipline, organization, use of health and hygiene, thus reducing waste and failures, improving the organizational climate, increasing employee productivity and motivation.

Keywords: Method 5S. Performance indicators. Processes Improvement

\section{INTRODUÇÃO}

O método " $5 \mathrm{~S}$ " é de uma vasta informação, com uma infinidade de pontos a serem abordados. Dentre desses pontos, esse artigo distingue o debate sobre como esse poderoso instrumento, pode ajudar com a organização, limpeza e bem-estar dos colaboradores. Esclarecer como o método " $5 \mathrm{~S}$ " adapta o fornecimento da distribuição de acordo com a demanda e como isso pode tornar ótimo um processo que pode estar desorganizado, trata-se das principais dificuldades desta pesquisa. Como o método pode garantir uma visão satisfatória do que e quando organizar e como isso contribui nos fluxos da empresa? Demonstrar na prática essa dinâmica do funcionamento do método " $5 \mathrm{~S}$ ", constitui o objetivo deste trabalho, alcançado através de pesquisa realizada em uma distribuidora de produtos de higiene profissional. Falcão et al. (2015) ressalva que a mudança e a quantidade de modelos e ferramentas evitam a assimilação do melhor modelo de gestão de qualidade adequada à realidade da empresa. Dessa forma, uma empresa que emprega o " $5 \mathrm{~S}$ " na administração dos seus processos, melhora suas chances de competitividade no mercado, uma vez que esse sistema favorece a redução de itens desnecessários, a eliminação de desperdícios e custo reduzido. Aqui se estabelece a relevância desse estudo, a justificativa deste trabalho.

A utilização do método " $5 \mathrm{~S}$ " na administração é um fator de ajuda para o controle da organização e redução no tempo de processo, que deve ser comprovado pelos dados obtidos na pesquisa descritiva realizada, juntamente com o material bibliográfico pesquisado e elaborado, que será apresentado nos capítulos que seguem. O trabalho está estruturado em capítulos e seções, sendo o primeiro a Introdução, onde consta a demarcação do assunto, o problema da pesquisa, o objetivo, a justificativa e a hipótese. O segundo capítulo relata a 


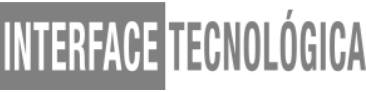

fundamentação teórica, falando de cada "S" e o que cada um representa. O procedimento metodológico é apresentado no terceiro capítulo, e na sequência o quarto capítulo, que relata com os resultados apresentados, obtidos através da pesquisa descritiva feita. Por fim, são apresentadas as considerações finais e as referências bibliográficas utilizadas na pesquisa.

\section{FUNDAMENTAÇÃO TEÓRICA}

\subsection{Método "5S"}

O método 5S surgiu no Japão quando o grupo do professor Kaoru Ishikawa lançou um método simples, porém eficiente, como princípio de reduzir os desperdícios e eliminar as perdas. Isto colaborou com o início de estratégias que envolvem à qualidade que ajudaram na transformação das empresas japonesas, levando a descoberta e obtendo sucesso na implementação do método $5 \mathrm{~S}$. O procedimento teve o destaque de alinhar padrões de qualidade com comportamentos dos funcionários (CARVALHO, 2011).

O método $5 \mathrm{~S}$ tem como finalidade envolver à manutenção da limpeza, controle de utilização e descarte, identificar e organizar o que precisa, padronizar as práticas saudáveis e colocar como habito a autodisciplina, envolvendo todas as pessoas desde o menor nível hierárquico ao topo (TRAINOTTI, 2007).

De acordo com Paladini (2012), a qualidade de uma organização é definida pela melhoria contínua de todas as suas tarefas, fazendo a mudança dos processos de produção. Abrantes (2009) afirma que com esse método, o Japão passou por cima dos problemas e dificuldades, superando expectativas de qualidade e produtividade, sendo usado como referência para as empresas.

\subsubsection{Seiri (senso de utilização)}

No senso de utilização, qualquer material do trabalho é avaliado (máquinas, equipamentos e burocracia), vendo o seu destaque para colocar em prática o trabalho, de tal maneira, que são as mais usadas fiquem mais juntas do funcionário e da atividade a ser feita. A principal função do Seiri é colocar em prática o menor índice de tarefas desnecessárias no trabalho. Segundo Trainotti (2007), na prática, durante a implantação desse primeiro senso as 


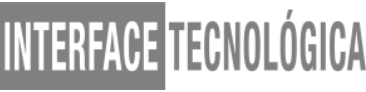

organizações promovem um evento diferente, paralisando um dia de trabalho, conhecido como o "Dia D." Dessa forma, a atividade não se torna empírica ou submetida a parâmetros subjetivos e pessoais

\subsubsection{Seiton (Senso de organização)}

Esse senso constitui em posicionar tudo aquilo que está fora de ordem em seu devido lugar, aceitando assim, uma rápida localização e acesso às mercadorias e objetos, evitando a perda de tempo. (LOBO, 2012). Segundo Osada (2010), o Seiton se destaca pelo fim da procura de objetos e ferramentas. Feito isso, depois que a organização é feita, é cumprida a qualidade, a segurança e agilidade no tempo. O senso remete à ordem e, a gestão, deve estipular locais acessíveis para armazenar documentos úteis, por exemplo, para encontrar de forma mais rápida possível. A ordenação deve ter o sentido de ter à mostra materiais, ferramentas e objetos de uma maneira organizada, estabelecendo um alto nível de um visual panorâmico para acessos rápidos e concisos.

\subsubsection{Seiso (Senso de limpeza)}

Para Lobo (2012), o senso de limpeza constitui em eliminar a sujeira, materiais e objetos que não fazem parte daquele ambiente. O mais importante mesmo é não sujar, descartando os dejetos em locais corretos. Osada (2010, p. 116) diz que a "limpeza é sinônimo de fiscalização". Para a gestão das empresas, é necessário atualizar os dados e as informações sobre conscientização ambiental, para que as tomadas de decisões sejam mais certeiras.

\subsubsection{Seiketsu (Senso de padronização)}

O senso de padronização mira as naturezas e adversidades que beneficiam a saúde física, mental e espiritual, a fim de cravar que o ambiente seja livre de poluentes, ter boas condições sanitárias, cuidar da higiene pessoal, mantendo os exames periódicos dos colaboradores em dia e fortalecer assim um ótimo vínculo profissional. Igualmente, é cuidar pelos dados e informações, para que estejam claros e precisos, de fácil interpretação e 


\section{WNERPHACETEENOLOGGCA}

entendimento, tudo isso relacionado à Seiketsu, que significa saúde e higiene (LOBO, 2012). É o destaque voltado para o gerenciamento visual e padrão, para manter condições favoráveis, fazendo com que se esteja sempre alinhado ao tudo o que acontece (OSADA, 2010). Seiketsu significa esmero e visa fortificar três outros sensos (utilização, organização e limpeza) para manter as condições de saúde, ficando livre de poluidores. Para a gestão da empresa, destaca que o senso tem como finalidade agregar, prever e formular informações e dados para serem de fácil absorção, sendo muito benéfico e prático para as determinações. O sentido deste senso, então, é ter todos os colaboradores desempenhando os processos de segurança e atentos com a sua saúde em sentido expansivo.

\subsubsection{Shitzuke (Senso da disciplina)}

Saber vigiar e cuidar pelas regras, normas e questões burocráticas é o que explica o Shitsuke, sendo bem importante que a pessoa cresça a partir da prática, mostrando a reverência por si e pelos outros (LOBO, 2012). Esse senso diz respeito a autodisciplina, tendo colaboradores empenhados, cumprindo rigorosas regras éticas, estratégicas e com melhorias ininterruptas a nível pessoal e grupal. Osada (2010, p. 155) distingue esse senso como a "disciplina como uma forma de alterar costumes". Se unir para a construção de uma equipe mais forte. "Quanto mais difícil é o serviço, mais fundamental é a união, pois até o mínimo erro pode levar ao insucesso".

\subsection{Indicadores de desempenho}

Para Caridade (2006) os indicadores de desempenho são demonstrados como sendo elementos e/ou informações, geralmente numéricos, que especificam um certo acontecimento e que são agregados para medir um processo e seus resultados, que podem ser adquiridos durante a sua realização ou ao seu término. Em suma, Parmenter (2010), cita que estes agem como um conjunto de proporção cujo foco geralmente é dado a itens do desempenho organizacional que são mais decisivos, do ponto de vista para o sucesso da organização.

KPI é a sigla em inglês para Key Performance Indicators, ou traduzindo para o português, Indicadores-Chave de Desempenho. Trata-se, na verdade, de uma ferramenta de gestão para medir o desempenho e o acontecimento de uma empresa (SHANA; 


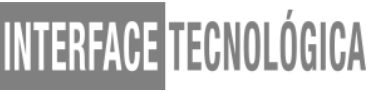

VENKATACHALAM, 2011). Por meio desse instrumento, é possível entender o que está trabalhando e o que não está, e fazer as alterações precisas para chegar aos objetivos estabelecidos. Os KPI's são indicadores que ajudarão os gestores a monitorar o desenvolvimento das operações, evitando assim a acumulação e a utilização de informações irrelevantes. Ou seja, um KPI é capaz de medir atividades cujo resultado é expressivo no desempenho futuro e influenciam os resultados das operações da organização (YIN et al., 2014). Outra função essencial dos KPI's é ajudar a transmissão da visão e da missão de uma empresa aos colaboradores que não estão em responsabilidades elevadas. Afinal, ao colocar e dividir os que vão medir o sucesso de um processo, deixa-se aberto para os colaboradores o que realmente importa na administração (ELMONGY et al., 2013), promovendo ainda a análise do desempenho da empresa.

\section{PROCEDIMENTOS METODOLÓGICOS}

Este trabalho desenvolvido constituiu de uma revisão bibliográfica, seguido de uma pesquisa descritiva realizada em uma distribuidora de produtos de higiene profissional, que mostra a importância de aplicar o método $5 \mathrm{~S}$ em um ambiente empresarial. A empresa está localizada no interior do Estado de São Paulo, mas, por questões de sigilo, não permitiu a divulgação de seu nome, sendo tratada no texto como "empresa em estudo".

A pesquisa descritiva visa o reconhecimento, o registro e estudo da interpretação das situações, fatores ou variantes que se relacionam com o fenômeno ou processo, de modo físico sem a influência do pesquisador-observador (PEROVANO, 2014).

O pesquisador tem a função de ressaltar todo o processo de um sistema, desde as suas funcionalidades até a sua estrutura, seja ele método ou uma realidade operacional, sem interferir nele.

A escolha das referências é feita através de livros, artigos, internet e revistas científicas, buscando assuntos em base do tema do trabalho escolhido, com os dados obtidos na pesquisa realizada pelo método Descritivo. 


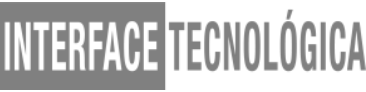

\subsection{Funcionamento da distribuidora de higiene profissional com o método "5s"}

A empresa onde realizou a pesquisa possui uma linha diversificada com mais de 200 produtos, atendendo vários segmentos de clientes como: hospitais, hotéis, clubes, escolas, cervejarias, indústrias alimentícias e empresas em geral. Nela, o método $5 \mathrm{~S}$ é aplicada para os setores de logística, planejamento e assessoria. Para que a empresa em estudo obtenha sucesso na aplicação do método, é preciso que se conheça como deve ser feita a implementação da mesma. Segundo Lobo (2012), em primeiro lugar, deve-se envolver todos os que fazem parte da empresa; num segundo momento, é importante dividir os espaços físicos, cada área mencionada deve formar uma comissão, com um líder no mínimo, para que seja implantada a sistemática $5 \mathrm{~S}$; já no terceiro passo, devem ser observados algumas prioridades:

- Layout - Um local para executar as tarefas, circular as pessoas, trafegar o maquinário, transportar matérias-primas;

- Mobiliário - patrimônio que pode ser utilizado para acomodar pessoas, guardar materiais, expor decorações ou protocolar arquivos;

- Dispositivos - É necessário aparelhos mecânicos e elétricos que sejam usados como complemento de trabalho;

- Documentos - São agregadas fontes de pesquisa, informações, dados, registros e estudos;

- Matéria-prima - Material necessário para elaborar atividades ou afazeres e preservar a equipe garantindo o seu conforto.

\section{RESULTADOS E DISCUSSÃO}

Após analisar a parte teórica, que traz os conteúdos sobre os cinco sensos e como aplicar, chega a vez de colocar em prática. Em primeiro lugar, os colaboradores da empresa em estudo foram orientados a colocar em prática os cinco sensos. O primeiro senso (Seiri Senso de utilização) usou-se com maior destaque e os demais foram ilustrados de uma forma geral. Durante algumas conversas com a empresa, foram abordados os principais motivos da escolha dessa sistemática; o que era necessário para aplicar, que trata da organização, com o descarte de materiais inutilizados. 


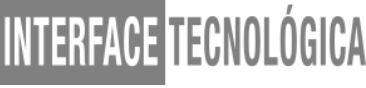

Ao início da aplicação do primeiro senso, a empresa viu que não seria fácil. Então, era necessário conversar e mostrar aos colaboradores o quanto os sensos são importantes e o quanto será vantajoso a aplicação deles. Será necessário ter ciência do que realmente precisa, a fim de eliminar o que não precisa. Então, formulou-se um desafio entre o gestor e os colaboradores da distribuidora a conversarem se realmente os materiais trabalhados seriam ou não utilizados, se poderiam ser usados num futuro próximo ou se poderiam ser descartados.

Questionou-se às equipes dos setores sobre a utilidade dos materiais dentro dos escritórios, logística e estoque. Acredita-se que essa aplicação se tornou interessante para auxiliar na obtenção de êxito no trabalho, uma vez que os gestores tomaram consciência da importância da sistemática na distribuidora. Depois de passar por esse processo, os materiais necessários foram reorganizados e os demais descartados, levando-os para empresas que dão o descarte correto ou diretamente ao lixo.

A área de telemarketing, modificada em alguns itens, consistiu em aplicar treinamentos de comunicação, clareza e persuasão com o objetivo de agilizar os atendimentos. Uma recomendação, para colocar em prática, é fazer uma semana de conscientização sobre a limpeza no setor e orientações sobre postura e ergonomia. Os funcionários do setor foram avaliados e escolhidos para fazerem dinâmicas, com os objetivos de gerar harmonia em equipe e integração.

Segundo Francischini (2017), os indicadores são ajustes qualitativos ou quantitativos que aparecem a situação de uma operação, processo ou sistema. Desempenho é a comparação do que foi alcançado pela operação em relação a uma espera do cliente ou meta do gestor. Logo, indicadores de desempenho são ajustadas que aparecem a comparação do que foi atingido pela operação em relação a uma experiência ou objetivo. Uma alteração importante em aderir ao método KPI (Key Perfomance Indicator), que revela indicadores de desempenho, é registrar o tempo de atendimento de cada funcionário. Dentre outros indicadores, usou-se o nível de satisfação do cliente, medido por pesquisar ao final da ligação a opinião do cliente de como recebeu o atendimento, o indicador TME (Tempo Médio de Espera) e a Taxa de Conversão (indicando por números ou porcentagem os sucessos obtidos nos atendimentos). E para terminar, uma outra ideia é sugerir vagas para recrutamento interno, como sinal de motivação e reconhecimento dos funcionários.

Os indicadores de gestão apresentam-se como utensílio para medição do nível de desempenho de processos na organização (MOHAMMED BADAWY et al., 2016), sendo 


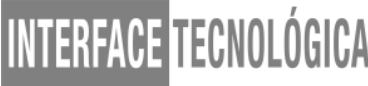

imprescindíveis para comparação do desempenho real com a meta estimada. Lobato et al., (2009) afirma que o avanço contínuo dos processos internos à empresa engloba a criação e utilização de indicadores de desempenho para servirem de base na estimativa dos resultados gerais, produtos, serviços de apoio e atividades. Takashina e Flores $(2005$, p. 3) comenta que os indicadores devem ser "ligados às áreas do negócio cujas execuções causam maior impacto no sucesso da organização. Desta maneira, eles dão apoio ao exame crítico dos resultados do negócio, às tomadas de decisão e ao replanejamento."

Houve mudanças na sala de gerência e na sala de reuniões, com a aquisição de um único armário para guardar arquivos. Foi aplicado o conceito de $5 \mathrm{~S}$ nas reuniões existentes, possibilitando a otimização das mesmas, visto que foi contabilizado que havia conflito entre os conteúdos e participantes. Para cada reunião foi elaborado um quadro de referência, além de serem registradas nas atas, definindo a frequência da realização da reunião, o tempo máximo, os participantes, a padronização das informações de entrada de cada reunião, bem como as informações que cada reunião deverá produzir, quais indicadores deverão ser tratados no momento, estabelecendo papeis e responsabilidades entre os participantes.

Os envolvidos das reuniões realizaram o inventário das pautas de cada reunião existente e aplicaram o Primeiro S (Seiri), identificando que conflitos e até em alguns casos reuniões distintas com pautas repetidas, ou pautas não prioritárias em relação ao planejamento estratégico da empresa. Aplicaram o Segundo S (Seiton), organizando a sequência que cada reunião deveria ser realizada, visto que alguns produtos de algumas reuniões eram entradas para outra, além da atenção do melhor local a ser realizada. O Terceiro S, realizaram a limpeza, dos temas abordados em algumas pautas e até mesmos alguns participantes, pois identificaram que na realidade não tinham poder de decisão e só deveriam receber a informação da decisão tomada na reunião. O quarto $\mathrm{S}$, foi a criação dos quadros de referência, ou seja, para cada reunião foi elaborado um quadro determinando a frequência da realização da reunião, tempo máximo, participantes, padronização das informações de entrada de cada reunião, bem como as informações que cada reunião deverá produzir, quais indicadores deverão ser tratados no momento, estabelecendo papeis e responsabilidades entre os participantes. O Quinto S aplicado foi a sistemática para controlar se as reuniões estão ocorrendo no momento, no tempo, com os participantes determinados e evidenciando que os indicadores estão sendo geridos. 


\section{WNERPHAETEECNOLOGGCA}

Dentre outras ideias sugeridas foram: eliminar livros contábeis antigos, formulários duplicados, por meio de uma limpeza profunda, organizar mesas, gavetas, armários e a área de trabalho do computador, separar ficheiros e pastas por uso de cores, etiquetas ou números, facilitando o manuseio. E para progredir o crescimento da empresa, é necessário investir no endomareting e estimular o trabalho em grupo, permitindo que a criatividade saia do papel.

Em outro momento, aplicou-se os " $5 \mathrm{~S}$ " na área de logística. No local, criou-se placas e etiquetas de identificação, indicando os locais onde cada material deverá ser guardado e onde eles estarão, caso alguém os procure, evitando a perda de tempo. Uma outra sugestão é sobre a verificação dos veículos, fazendo reparos preventivos, a cada 10 mil km rodados. Colocouse em prática o planejamento da rota no dia a dia, evitando viagens desnecessárias e fazendo um check-list dos produtos a serem despachados antes de começar a viagem.

Avaliou-se o estoque de materiais, onde visto a utilidade de cada objeto e descartando o que é desnecessário, como paletes quebrados, por exemplo. Foram inseridos inventários de forma periódica, sinalização de locais onde pode ocorrer acidentes de trabalho e a adoção do sistema de classificação de etiquetagem, que facilite visualizar produtos que estão para vencer e produtos que podem acabar o estoque.

Após a aplicação do 5S na área logística, foi implementado os indicadores de Tempo Médio de Permanência de Veículo (TMPV), que possibilita gerir o tempo de entrada e saída dos veículos tanto para descarregamento de produtos quanto para carregamento das vendas, assim dimensionando a produtividade necessária e o número de funcionários.

Foi possível comprovar que a organização do layout e a definição dos posicionamentos dos materiais no almoxarifado proporcionaram a redução do TMPV e consequentemente o aumento da produtividade da equipe de recebimento e expedição, reduzindo o custo de hora-extra e melhorando o nível de atendimento ao serviço.

Outro ponto observado foi a melhoria da acuracidade do carregamento e a redução da reclamação por erro de carregamento (falta, excesso ou erro de produto entregue ao cliente).

\section{CONSIDERAÇÕES FINAIS}

O objetivo deste trabalho é demonstrar na prática o funcionamento do método " $5 \mathrm{~S}$ ", no setor de estoque, logística, recepção, telemarketing e salas administrativas. Dentro do conceito, o objetivo deste trabalho alcançou as expectativas, com a conferência dos elementos 


\section{WNERPHAETEECNOLOGGCA}

levantados na pesquisa realizada dentro de uma distribuidora de produtos de higiene profissional que entrega produtos de limpeza e resíduos de hospitais, hotéis e clubes. Ao longo de todo o estudo, foram apresentados conceitos e definições acerca do método " $5 \mathrm{~S}$ ", colaborando assim para eliminar desperdícios, reduzir custos, desertar ideias, reduzir tempo desnecessário. Conforme dados apresentados na pesquisa descritiva, a empresa atua em um sistema de distribuição regional, com estoque adequado, com baixo risco de fornecer de maneira sem sucesso. A administração da empresa proporciona agilidade no fluxo do processo de vendas e logística, com baixa margem de erro na movimentação dos itens na distribuição.

O método apresentou melhora nos processos envolvidos e consequentemente nos indicadores de tempo médio de espera em $12 \%$, o tempo médio do atendimento em $6 \%$, proporcionando ao cliente o impacto médio da redução do tempo médio de atendimento em $18 \%$. O tempo médio de permanência de veículo reduziu $15 \%$ e a quantidade de horas extras semanais reduziu $8 \%$, devido ao aumento da produtividade da equipe de carregamento e descarregamento. O número de reclamações por atendimento, atraso de carregamento, erro de carregamento reduziu $13 \%$ e apresentou a redução por meio das entregas.

Com base na fundamentação teórica deste artigo, a pesquisa descritiva mostra que trabalhar cumprindo com a demanda, operando com estoque reduzido, eliminando desperdícios e custos nos estágios de cada processo envolvido, dentro do método " $5 \mathrm{~S}$ ", colabora e muito nos processos e impacta positivamente no lucro da empresa, se tornando assim mais competitiva.

\section{REFERÊNCIAS}

ABRANTES, José. Gestão da Qualidade. Rio de Janeiro: Interciencia, 2009.

CARIDADE, A.; Práticas de Gestão Estratégica e Aderência ao Método Sigma: Um estudo de Caso no Setor de Celulose e Papel. São Paulo: USP, 2006.

CARVALHO, P. C. O programa 5S e a qualidade total. Campinas, SP: Editora Alínea, $5^{\circ}$ edição, 2011.

EL-MONGY, A. M. A.; HAMOUDA, A. E.; NOUNOU, A.A.N. Wahdan Design of prediction system for key performance indicators in balanced scorecard. International Journal of Computer Applications, v. 72, n. 8. 2013. 
FALCÃO, A. et al. Uso da Metodologia 5s numa incubadora de empresas de base tecnológica de Campos dos Goytacazes-RJ. In: SIMPÓSIO DE ENGENHARIA DE PRODUÇÃO - SIMEP. 2015, João Pessoa. Anais. Paraíba, 2015.16p.

FRANCISCHINI, Paulino G. Indicadores de desempenho dos objetivos à ação métodos para elaborar KPIs e obter resultados. Rio de Janeiro: Altas Books, 2017. 448 p.

LEONEL, J. O programa 5s e sua aplicação em uma fábrica de embalagens de papel. 2011. 60p. Monografia (Bacharelado) -Faculdade de Engenharia da Universidade Federal de Juiz de Fora, Juiz de Fora, 2011.

LOBATO, D. M.; FILHO, J. M.; TORRES, M. C. S.; RODRIGUES, M. R. A. Estratégia de Empresas, 9 ed. Rio de Janeiro: FGV Management, 2009 2012.

LOBO, Renato Nogueirol. Gestão da qualidade. 1. ed. 3. reimpr. São Paulo: Érica,

MOHAMMED BADAWY, A. A.; EL-AZIZ, A. B. D.; IDRESS, A. M.; HEFNY, H.; HOSSAM, S. A survey on exploring key performance indicators. Future Computing and Informatics Journal, v. 1, n. 1 - 2, 2016.

OSADA, Takashi - Housekeeping, 5S's: Seiri, Seiton, Seiso, Seiketsu, Shitsuke. 4. ed. São Paulo: Instituto IMAM, 2010. 2012.

PALADINI E. P. Gestão da qualidade: teoria e prática. $3^{\text {a }}$ ed. Rio de Janeiro: Atlas,

PARMENTER, D. Key performance indicators: developing, implementing, and using winning KPIs. John Wiley \& Sons. 2010.

PEROVANO, Dalton Gean. Manual de Metodologia Científica. Paraná: Editora Juruá, 2014.

SHANA, J.; VENKATACHALAM, T. Identifying key performance indicators and predicting the result from student data. International Journal of Computer Applications, v. 25 , n. 9 , p. 45-48. 2011.

TAKASHINA, N.T.; FLORES, M.C.X. Indicadores de qualidade e do desempenho: Como estabelecer metas e medir resultados. Rio de Janeiro, Quality mark, 2005.

TRAINOTTI, M. S. Aplicação da Metodologia 5S, visando a melhoria do processo organizacional em uma Fundação de Direito Privado: Um estudo de Caso. 2007. 119 f. Dissertação (Mestrado em Engenharia Mecânica) - Programa de Pós-Graduação em Engenharia Mecânica - Departamento de Engenharia de Fabricação. Universidade Estadual de Campinas. Campinas, 2007. 
YIN, S.; WANG, G.; YANG, X. Robust PLS approach for KPI-related prediction and diagnosis against outliers and missing data. International Journal of Systems Science, v. $45, \mathrm{n} \quad$. 7, p. 1375-1382. 2014. 